\title{
Additive Manufacturing in Vascular Stent Fabrication
}

\author{
Yang Lei ${ }^{1,2, \#}$, Xin Chen ${ }^{1,2,3,4 \#}$, Lei Zhang ${ }^{1,2, a}$, Lei Li ${ }^{5}$, Shuangzhu Kang ${ }^{1,2}$, Chengjin Wang ${ }^{1,2}$ and Wei
Sun $^{1,2,6}$ \\ 1 Department of Mechanical Engineering, Tsinghua University, 100084, China \\ 2111 'Biomanufacturing and Engineering Living Systems' Innovation International Talents Base, 100084, Beijing, \\ China \\ 3 Ministry of Industry and Information Technology Equipment Industry Development Center, Beijing, 100846, China \\ China Center for Information Industry Development, Beijing, 100048, China \\ Beijing Huaxin Hospital First Hospital of Tsinghua University, Beijing, 100016, China \\ 6 Department of Mechanical Engineering, Drexel University, Philadelphia, PA, United States of America \\ \# These authors contributed equally to this work.
}

\begin{abstract}
High-efficiency formation of personalized stent by additive manufacturing (3D printing) has gained deal of attention and research in interventional and personalized medicine. In this article, the structural characteristics of vascular scaffolds and the application and innovation of additive manufacturing technology in the process of angioplasty are reviewed. In the future, with the continuous maturity of additive manufacturing technology, it is expected to be an important part of interventional precision medicine to manufacture personalized vascular stent.
\end{abstract}

\section{Introduction}

Vascular trauma and defect, as well as cardiovascular and cerebrovascular diseases, have become the main threats to human life and health. Due to the good performance on reconstruction of vascular structure, stents implantation has become the main treatment for vascular diseases[1]. Based on different fabricate structures, vascular stents can be divided into dense mesh stents and stent grafts. Dense mesh stents[2] include bare metal stents (BMS), drug eluting stents (DES) and bioresorbable stents (BRS) [3, 4]. After expanded, such stents can support blood vessels, while be fixed by the pressure produced by the vessel wall, in which case, the lumen unobstructed will be guaranteed. Dense mesh stents are used to treat coronary heart disease, ischemic stroke, peripheral vascular stenosis, etc. [5] Stent graft is a type of vascular stent with a fabric coat, it is expandable like bare metal stents, and the film creates a contained tube to replace the vascular wall. It is mainly used to repair aortic dissection, true aortic aneurysm, pseudoaneurysm, aortic penetrating ulcer and other diseases. It is also used in the treatment of intracranial aneurysms[6].

The current manufacturing methods of vascular stents are standardized, that is, the diameter and length of stents are serialized or standardized. However, there are individual differences in the diameter and shape of real blood vessels in the human body, which makes standardized stents difficult to match the personalized needs. If the stent size is not consistent with the patient's real vascular size after implantation, the therapeutic effect will be affected. Too small diameter of the stent will lead to poor

\footnotetext{
${ }^{a}$ Corresponding author : stoneszhang@mail.tsinghua.edu.cn
} 
adherence to the wall of the stent, resulting in internal leakage or displacement. Too large diameter can rupture blood vessels, leading to treatment failure. At the same time, the shape of real blood vessels in the human body is curved, therefore, some standardized stents may "shrivelled" after implantation, because they cannot adapt to the physiological structure of blood vessels. Therefore, the individualized customization of vascular stent is required in clinic [7-10].

Additive manufacturing (3D printing) can not only achieve the personalized vascular stent in a short time, but also avoid the treatment failure caused by the poor compliance of the traditional stent. In addition, compared with other personalized fabricate technology, the 3D printing efficiency of vascular stents is higher. Now, with the maturity of the additive manufacture technology, it has gained widely concern in the field of vascular stent fabrication and shown a good application prospect in the clinical treatment.

In this paper, the structural characteristics of vascular scaffolds are reviewed, as well as the status and prospect of the additive manufacture technology in vascular stent fabrication.

\section{Dense mesh stents}

\subsection{Structural features of dense mesh stents}

The dense mesh stent is an expanded mesh structure that provides sustained support in order to open a narrow occlusion vessel. It is mainly used for the treatment of cardiovascular and cerebrovascular diseases that are serious threats to human health such as coronary heart disease and stroke. With the gradual improvement of product performance, endovascular stents have undergone three development stages: BMSs, DESs and BRSs[11].

\subsubsection{Bare metal stents}

In 1964, Dotter et al. first proposed the concept of endovascular stent. In 1987, Swiss doctor Ulrich Sigwart[12] first implanted a metal stent into a coronary artery to support a stenotic obstruction vessel, so that the stent can reduce the elastic retraction of the blood vessel in order to cure a coronary heart disease. Since then, endovascular stents have been widely used in interventional therapy. The materials of BMSs is generally medical 316L stainless steel, titanium alloy, cobalt alloy, tantalum metal, etc., which have strong supporting force[13]. However, the traditional permanent metal stent will inevitably cause immunological rejection after implantation, which is easy to cause intimal hyperplasia, stimulate the proliferation of smooth muscle cells and cause postoperative restenosis, which seriously affects the long-term patency rate of the vascular stent[14]. Clinical statistics show that after bare metal stent implantation, the rate of restenosis reaches $20 \%$.

\subsubsection{Drug eluting stents}

DES is developed based on BMS. DES is mainly composed of a metal stent, a drug carrier and a drug coating. DES can inhibit the proliferation of vascular endothelial cells, the aggregation of plasma proteins and white blood cells, and prevent the restenosis of blood vessels through slowly release of drugs in the blood vessels[15]. DES has been developed by two generations. The first generation is coated with rapamycin or paclitaxel on a stent of medical stainless steel. The most representative products are Cypher (Figure 1.A) by Johnson\&Johnson[16] and Taxus (Figure 1.B) by Boston Scientific[17]. Since these drugs are prone to leukocytes and coagulation factors attached to the scaffold, the incidence of late stent thrombosis in the first-generation DES is significantly higher than that in BMS[18]. The second-generation DES is represented by the Abbott XienceV (Figure 1.C)[19], using cobalt-chromium alloy carry zotarolimus or eromox. The rate of restenosis is almost the same as that of the first-generation DES, but the incidence of late-stage stent thrombosis is significantly reduced[20]. 


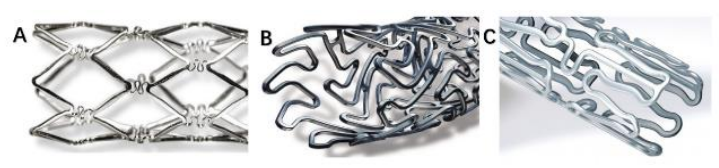

Figure 1. 1st \& 2ed generation DESs. (A) Cypher by Johnson\&Johnson; (B) Taxus by Boston Scientific; (C) XienceV by Abbott

\subsubsection{Bioresorbable stent}

The clinical data shows that the revascularization can be completed within 6-9 months after the implantation. At that time, as a foreign substance in vivo, permanent stent will hinder the recovery of vascular function, so the bioresorbable stent becomes a new research hotspot. BRSs can be divided into metal stents and polymer stents. The materials of the BRSs are mainly iron-based alloys, magnesium-based alloys and zinc-based alloys[21]. These metals are essential trace elements in human body and have good biocompatibility. However, how to match the degradation rate and the rate of blood vessel regeneration is a problem that metal bioresorbable stents need to solve. The polymer scaffold is generally made of a mixture or copolymer of poly-L-lactide (PLLA), polyglycolic acid (PGA), and polycaprolactone (PCL) [22-28]. In 2006, Abbott obtained the Guidant DES line from Boston Scientific for $\$ 6.4$ billion. Based on the Guidant BVS, polylactic acid BRS from Abbott obtained CE certification. In 2016, the Abbott Absorb (Figure 2)[29] was approved by the FDA for interventional treatment of coronary artery disease, which is the first degradable vascular stent in clinical use in the United States.

Figure 2. Abbott Absorb BRS.

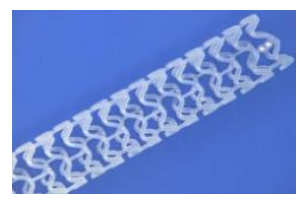

\subsection{Additive manufacturing of dense mesh stents}

In the research of vascular scaffold materials, the molding process has experienced the stages of filament winding, photochemical etching, injection molding and laser cutting[30]. In the early stage, stainless steel stents were fabricated by wire winding, and each unit was connected by laser local welding. However, the small size and complex structure of vascular stent result in inability to locate at the weld. The photochemical etching process avoids the positioning process, it uses ultraviolet light to etch the plane model of the vascular stent on the stainless steel sheet, which is then folded and welded into shape. However, the mesh structure processed by this technology is relatively soft, and it is easy to break in the process of crimping forming, while the welding points are arranged on one side of the stent, which affects the mechanical properties[31]. Injection molding preparation vascular stent process does not have above problems, but the vascular stent has high requirements for the injection molds. Moreover, the molding unevenness during the injection molding process is serious. Laser cutting technology has become a commonly used vascular stent processing technology because of its high processing precision and no change in material physicochemical properties[32]. However, toxic gases are generated during laser engraving[33], and the stent support section is rectangular, which may cause local eddy currents in the blood, which induce leukocyte aggregation and lead to restenosis[34].

These years, additive manufacturing technology has been concerned in vascular stent manufacturing. Since the formation of metal materials requires consideration of oxidation and other issues, the current additive manufacturing vascular stents are concentrated in the bioresorbable polymer stents.

In 2013, Flege et al.[35] of Aachen University of Technology, Germany used PLLA and PCL materials respectively to process BRSs using laser selective melting technology (SLM) for the first time, and pointed out that SLM technology has a good prospect in the manufacture of BRSs. However, the surface accuracy of the stent produced by this method is not high (Figure 3.A), which limits its 
development. In 2015, Park et al.[36] of the Korea Institute of Machinery and Materials used bio-cutting technology to manufacture a bioresorbable sirolimus-eluting PCL stent, but this technique makes it difficult to personalize the stent. In addition, the stents manufacture process by Flege and Park take more than $10 \mathrm{~h}$.

In 2015, Tumbleston et al.[37] of Carbon3D, U.S. developed continuous liquid interface production (CLIP) technology. The principle of CLIP: The UV projection solidifies the photosensitive resin, while the oxygen inhibits the solidification. The liquid resin at the bottom of the tank maintains a stable liquid area due to the contact with oxygen, thus ensuring the continuity of the solidification. This technology not only increases the speed of 3D printing by 25 to 100 times, but also make the layering infinitely delicate, which solves the problem of low precision of product surface. This technology plays a vital role in the rapid prototyping of high quality intravascular stents.

In 2016, van Lith et al.[38] of Northwestern University, U.S. synthesized a new citrate-based degradable polymer material, using micro CLIP technology to create customizable individualized arrow types. This stent (Figure 3.B) has good elasticity, strength, oxidation resistance and biodegradability, and the micro CLIP technology compresses the stent forming time to 70 minutes. According to the radial compression test, the maximum supporting force of $0.1 \mathrm{n} / \mathrm{mm}$ can be provided when the bracket is compressed by $25 \%$ in the radial direction. The stent was degraded by about $25 \%$ after 180 days. Although the parameters of the stent are relatively good, in fact, the mechanical properties of the stent cannot match the BRSs under clinical tests. In addition, the materials used in the stents are not FDA-approved. Although it is claimed that the modified material has good biocompatibility, there is still much work to be done before clinic test.

In 2017, Ware et al.[39] of Northwestern University, U.S. used bioresorbable photopolymerizable biomaterials as raw materials to print customizable BRSs with micro CLIP. This stent meet the micron-scale precision biomedical devices requirement. Compared to standard stereo lithography apparatus (SLA) technology, micro CLIP can reduce manufacturing time from hours to minutes. At the same time, stents produced by the micro CLIP has better surface finish and more uniform mechanical properties. The mechanical properties of micro CLIP stents can match Nitinol stents. After optimization, a $2 \mathrm{~cm}$ high vascular stent containing 4000 layers can be fabricated in 26.5 minutes.

Also in 2017, Cabrera et al.[40] of Eindhoven University of Technology, Netherlands printed the vascular stent using fused deposition modeling (FDM) technology. The printing device is BakerBot by Replicator, and the printed material is thermoplastic copolyester elastomer (TPC). Mechanical properties and differential scanning calorimetry (DSC) characteristics of the stent were tested, while, animal experiments showed that the stent was completely fused with the vessel wall within 8-16 weeks (Figure 3.C).

In 2018, Guerra et al.[41] of University of Girona, Spain printed the PCL vascular stent (Figure 3.D) using the FDM technology. The relationship between the thickness of the stent and the temperature of the nozzle as well as the printing speed and the nozzle moving speed was summarized by a large number of experiments. Although the stent in this article did not achieve high surface resolution, it explored the $3 \mathrm{D}$ printing process in vascular stents fabrication.

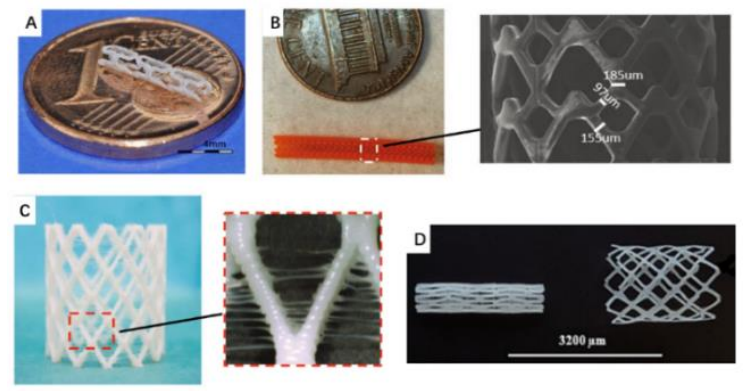

Figure 3. BRSs made by 3D printing technology. (A) BRS fabricated by Flege et al.; (B) BRS fabricated by van Lith et al.; (C) BRS fabricated by Cabrera et al.; (D) BRS fabricated by Guerra et al. 
In China, Liu Yuanyuan et al.[42] of Shanghai University used a combination of bio-3D printing and electrospinning technology to form a poly(p-dioxanone) (PPDO) slide buckle stent. The team used PPDO material to prepare the inner layer of the stent by 3D printing and prepared a mixture of chitosan and poly(vinyl alcohol) (PVA) to form the outer layer of the stent by electrospinning, and planted cells on the stent. The results showed that the adhesion and proliferation of cells on stents were good because the outer layer of the stents contained chitosan, a natural biological material. This article provides a new idea for 3D-printed drug eluting and cell loading stents fabrication. Tangdu Hospital[43], Xian, successfully used the additive manufacturing technology to prepare the personalized titanium alloy porous venous extravascular stent, and completed the world's first additive manufacture extravascular stent implantation for nutcracker syndrome. Liu Qing et al.[44] developed a method for the preparation of a three-dimensional porous tubular scaffolds and equipment using additive manufacture. Lai Xuecong et al.[45] developed the additive manufacturing equipment for the preparation of cardiovascular scaffolds, which can be printed and shaped on the rotating axis, providing a new method for the preparation of vascular scaffolds.

In 2018, Wei Sun et al.[46] of Tsinghua University, China developed a 4-axis linkage 3D printing platform based on the FDM technology. In the platform an extra axis, rotating mandrel structure, is added to avoid the support structure, and developed a set of mini-screw extrusion nozzle. The nozzle can use granular biological material, and the extruded wire diameter is $100 \mu \mathrm{m}$, which matches the requirements of the endovascular stent. The control model of the start-stop process of the screw nozzle was established to solve the start-stop delay control problem. At the same time, the effects of quenching and high-speed centrifugation on the mechanical properties of the stent were studied using PCL and PLLA materials, and the mechanical properties of the stent were optimized (Figure 4).

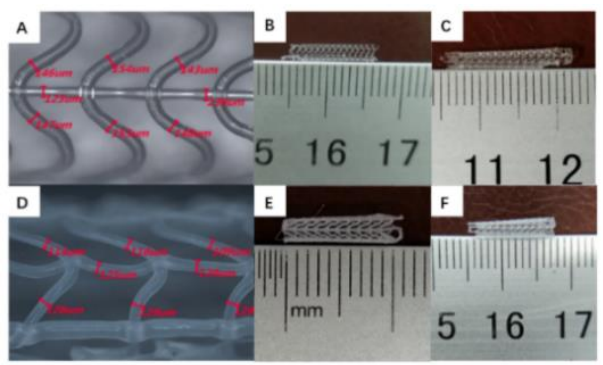

Figure 4. BRSs fabricated by Wei Sun et al., Tsinghua University. (A)Micrograph of PLLA stent; (B)Cylinder PLLA stent; (C)Conical PLLA stent; (D)Micrograph of PCL stent; (E)Cylindrical PCL stent; (F)Conical PCL stent.

\section{Stent graft}

\subsection{Structural features of the stent graft}

The stent graft is mainly used in endovascular aneurysm repair (EVAR), which has been widely used as an effective means for the treatment of descending aortic disease, aneurysm and other diseases[47]. EVAR is using a stent graft to replace the vascular wall to prevent blood entering the vascular false lumen[48]. In this way, the false lumen can be prevented from further expanding and rupturing to achieve the purpose of treatment.

The stent graft consists of a membrane and a metal stent. The film is generally made of polymer materials such as polyethylene terephthalate (PET), polytetrafluoroethylene (e-PTFE), and polyester[49]. There are also precedents for using silk as a film in China. The metal stents material are partially stainless steel, cobalt-chromium alloy and nickel-titanium alloy according to the different surgical release methods. At present, stent grafts that have been used in clinical practice are mainly produced and sold by several companies such as Medtronic, Cook, Jotec and Relay. The products use a wave or $\mathrm{Z}$ structure to support the blood vessels. The metal stent material is Nitinol so that self-expansion can be achieved. The film material is mainly PET and e-PTFE (Table 1). 
Table 1. Stent Grafts on sale

\begin{tabular}{|c|c|c|c|}
\hline Brand & Structure & Film Material & Metal Material \\
\hline Medtronic & wave & PET & Nitinol \\
\hline Jotec & wave & e-PTFE & Nitinol \\
\hline Relay & wave & e-PTFE & Nitinol \\
\hline Boston Scientific & wave & PTFE & Nitinol \\
\hline Cook & $Z$ & e-PTFE & stainless steel \\
\hline
\end{tabular}

\subsection{Additive manufacturing of stent graft}

At present, the mainstream stent grafts on the market include two parts: a polymer film and a nitinol stent. The forming process of Nitinol includes laser cutting, weaving, etc., which is similar to BMSs forming process[50]. While the film forming process is mainly divided into: textile forming and others. The textile forming method is to use the loom to weave polyester fiber into a tubular film. Because of its compact structure, stable size and controllable thickness, it is widely used by manufacturers of stent graft[51]. Other forming methods mainly include extrusion forming, electrostatic spinning, micro-nano composition, etc., to form PET or e-PTFE materials[52]. Medal stent and film binding methods include sewing, weaving, etc.[53]

Due to the difficulty in integrating and directly forming the film and the metal stent material, Lei Zhang et al.[54] of Tsinghua University proposed rapid prototyping sacrificial core-coating forming (RPSC-CF) technique. 3D printing is used to form a water-soluble, non-biotoxic core to ensure the inner cavity structure of the stent. Next, one or more material deposition processes, such as infiltration coating, spraying, electrostatic copy wire, are adopted to form multi-layer pipe wall structure by layer depositing materials. Finally, the inner core was immersed to obtain the stent. RPSC-CF has successfully prepared curved and bifurcation stent grafts to meet the real anatomical structure of the thoracic descending aorta. Meanwhile, the thickness of the film is controllable, which provides a basis for the patient's personalized use. The preparation cycle is about 3-4 days, and with the continuous adjustment of the preparation process, the entire stent graft fabrication is expected to be compressed within 48 hours, which can meet the treatment timing of patients with acute aortic dissection (Figure 5).

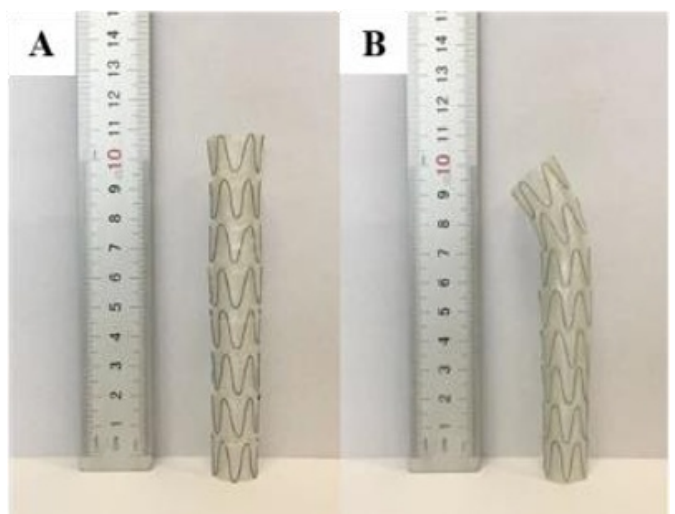

Figure 5. Stent grafts fabricated by Lei Zhang et al., Tsinghua University. (A)Straight type stent graft; (B) Curved stent graft.

\section{Conclusion}

Although the current vascular stent additive manufacturing technology is still in the research and development stage, the additive manufacturing technology can complete personalized stents in a short 
time, at the same time, avoid the treatment failure due to poor adherence and compliance. Compared with other personalized stent manufacturing processes, it has a higher application prospect in the field of clinical treatment.

With the development of precision medicine, personalized vascular stents that conform to the patient's true vascular structure have become the purpose of the next generation stents. Additive manufacturing of vascular stents has broad application prospects in the field of preparation of implantable medical devices due to its advantages of individualization and rapid prototyping. Additive manufacturing vascular stents can custom-make parameters of the stent shape, material, thickness, radial strength, etc. In 2018, Tsinghua University first packaged the biosensor on the 3D printing personalized stent graft. After the implantation, it could monitor the intravascular physiological signal in real time, track the working state of the stent stably, and provide precision treatment through data analysis. The program realizes the intelligence of the vascular stent production and provides a new idea for the development of the implantable medical device. It is believed that in the near future, a new generation of vascular stents combined with individualization, digitization, intelligence and networking will appear and provide precise medical treatment for patients with cardiovascular and cerebrovascular diseases.

\section{Acknowledgements}

Authors would like to thank Beijing Municipal Health Planning Commission (2016-2-4121), Beijing Life Science Frontier Technology Cultivation Project (Z141100000214012) and Natural Science Foundation of Beijing Municipality (Z150001).

\section{Reference}

1. Fuster, V., Top 10 cardiovascular therapies and interventions for the next decade. Nature Reviews Cardiology, 2014. 11(11): p. 671-683.

2. Li, M., A novel technique of endovascular treatment for the cerebral aneurysm: the development of covered stent specially designed for intracranial vasculature. Journal of Interventional Radiology, 2010. 19(4): p. 253-256.

3. Guo, Y. and Z. Tan, Features of endovascular stent materials and their therapeutic effects on ischemic stroke. Journal of Clinical Rehabilitative Tissue Engineering Research, 2013. 17(12): p. 2257-2264.

4. Mei, M., Characteristics and clinical application of different types of cerebral vascular scaffolds. Journal of Clinical Rehabilitative Tissue Engineering Research, 2014.

5. Liu, D., Q. Luo, and Z. Chang, Current Status and Progress of Minimally Invasive Medical Devices. Chinese Journal of Medical Instrumentation, 2008. 14(3): p. 2-11.

6. Liu, D. and T. Xi, Current Situation and Development Tendency of Interventional Medical Device\&Material Industry. Chinese Journal of Medical Instrumentation, 2006. 12(12): p. 1-14.

7. White, G.H., et al., Endoleak as a complication of endoluminal grafting of abdominal aortic aneurysms: classification, incidence, diagnosis, and management. Journal of Endovascular Surgery the Official Journal of the International Society for Endovascular Surgery, 1997. 4(2): p. 152.

8. Coppi, G., et al., Experience with the Stentor endograft at four Italian centers. J Endovasc Surg, 1998. 5(3): p. 206-215.

9. Roos, J.E., et al., Detection of endograft fractures with multidetector row computed tomography. Journal of Vascular Surgery, 2005. 42(5): p. 1002-1006.

10. Junnarkar, S.P., et al., Endovascular repair for acute symptomatic and ruptured abdominal aortic aneurysms. Ulster Medical Journal, 2001. 70(1): p. 51-53.

11. Duda, S.H., et al., Physical Properties of Endovascular Stents: An Experimental Comparison. Journal of Vascular \& Interventional Radiology, 2000. 11(5): p. 645-654.

12. Sigwart, U., et al., Intravascular stents to prevent occlusion and restenosis after transluminal angioplasty. N Engl J Med, 1987. 316(12): p. 701-706. 
13. M, F., et al., A new austenitic stainless steel with negligible nickel content: an in vitro and in vivo comparative investigation. Biomaterials, 2003. 24(27): p. 4929-4939.

14. Jr, H.D., In-stent restenosis. Reviews in Cardiovascular Medicine, 2001. 2(3): p. 115-119.

15. Pfisterer, M., et al., Late clinical events after clopidogrel discontinuation may limit the benefit of drug-eluting stents: an observational study of drug-eluting versus bare-metal stents. Journal of the American College of Cardiology, 2006. 48(12): p. 2584-2591.

16. Johnson, J. CYPHERTM Sirolimus-eluting Coronary Stent on RAPTORTM Over-the-Wire Delivery System. 2003; Available from: https://www.accessdata.fda.gov/cdrh_docs/pdf2/p020026c.pdf.

17. Scientific, B. TAXUS Liberte and TAXUS Liberte Atom. 2008; Available from: https://www.accessdata.fda.gov/cdrh_docs/pdf6/p060008s008c.pdf.

18. Mauri, L., et al., Stent thrombosis in randomized clinical trials of drug-eluting stents. N Engl J Med, 2007. 356(10): p. 1020-1029.

19. Abbott. The XIENCEV Everolimus Eluting Coronary Stent System Instructions for Use. 2008; Available from: https://www.accessdata.fda.gov/cdrh_docs/pdf7/p070015c.pdf.

20. Rasmussen, K., et al., Efficacy and safety of zotarolimus-eluting and sirolimus-eluting coronary stents in routine clinical care (SORT OUT III): a randomised controlled superiority trial. Lancet, 2010. 375(9720): p. 1090-1099.

21. Dave, B., Bioresorbable Scaffolds: Current Evidences in the Treatment of Coronary Artery Disease. Journal of Clinical \& Diagnostic Research Jcdr, 2016. 10(10): p. OE01.

22. Caiazzo, G., et al., Absorb bioresorbable vascular scaffold: What have we learned after 5 years of clinical experience? International Journal of Cardiology, 2015. 201: p. 129-136.

23. Shrivastava, S., Medical device materials. Advanced Materials \& Processes, 2004.

24. Moliterno, D.J., Healing Achilles--sirolimus versus paclitaxel. New England Journal of Medicine, 2005. 353(7): p. 724-7.

25. Huang, M.H., S. Li, and M. Vert, Synthesis and degradation of PLA-PCL-PLA triblock copolymer prepared by successive polymerization of $\varepsilon$-caprolactone and -lactide. Polymer, 2004. 45(26): p. 8675-8681.

26. Jiang, w., et al., Application of L-lactic acid and its derivatives in food industry. Journal of Food

27. Tsuji, H., Poly(l-Lactide). 2015: Springer Berlin Heidelberg.

28. Bährlerapp, M., Springer Lexikon Kosmetik und Körperpflege. 2007, Springer Berlin Heidelberg.

29. Abbott. Absorb GT1 ${ }^{\mathrm{TM}}$ Bioresorbable Vascular Scaffold (BVS) System 2016; Available from: https://www.accessdata.fda.gov/cdrh_docs/pdf15/p150023b.pdf.

30. Dun, S., Study on Micro Injection Molding Technology of Biodegradable Stent. 2013, Dalian University of Technology.

31. Huang, B., et al., Fabricating polymer stents with injection molding. 2008, US.

32. Yu, J.T., et al., Additive Manufacturing of Patient-Customizable Scaffolds for Tubular Tissues Using the Melt-Drawing Method. Materials, 2016. 9(11): p. 893.

33. T, Y., et al., Bifurcation lesions: two stents versus one stent--immediate and follow-up results. Journal of the American College of Cardiology, 2000. 35(5): p. 1145-1151.

34. Peng, H., The Numerical Analysis on the Influence of Hemodynamic to Different Cross-sections of Struts. Journal of Biomedical Engineering Research, 2013(3): p. 158-161.

35. Flege, C., et al., Development and characterization of a coronary polylactic acid stent prototype generated by selective laser melting. Journal of Materials Science Materials in Medicine, 2013. 24(1): p. 241-255.

36. $\mathrm{Su}$, A.P., et al., In vivo evaluation and characterization of a bio-absorbable drug-coated stent fabricated using a 3D-printing system. Materials Letters, 2015. 141: p. 355-358.

37. Tumbleston, J.R., et al., Additive manufacturing. Continuous liquid interface production of 3D objects. Science, 2015. 347(6228): p. 1349-1352.

38. Van Lith, R., et al., 3D-Printing Strong High-Resolution Antioxidant Bioresorbable Vascular Stents. Advanced Materials Technologies, 2017. 1(9): p. 1600138.

39. Ware, H.O.T. and C. Sun. Process development for high-resolution 3D-printing of bioresorbable vascular stents. in SPIE OPTO. 2017. 
40. Cabrera, M.M., et al., Computationally designed 3D printed self-expandable polymer stents with biodegradation capacity for minimally invasive heart valve implantation:A proof-of-concept study. 2017.

41. Guerra, A.J. and J. Ciurana, 3D-printed bioabsordable polycaprolactone stent: The effect of process parameters on its physical features. Materials \& Design, 2017. 137.

42. Liu, Y., et al., Composite bioabsorbable vascular stents via 3D bio-printing and electrospinning for treating stenotic vessels. Journal of Southeast University, 2015(2): p. 254-258.

43. Tangdu Hospital completed the world's first 3D printed extravascular stent implantation for Nutcracker Syndrome. Journal of The Fourth Military Medical University, 2016(1): p. 4-4.

44. Liu, Q., Z. Wang, and Y. Liu, Method for the preparation of a three-dimensional porous tubular scaffolds and equipment. 2015: $\mathrm{CN}$.

45. $\mathrm{Xu}, \mathrm{M}$., et al., Fast light curing nozzle for 3D printing equipment. 2015: CN.

46. Kang, S., Research on 3D printing equipment and forming technology of personalized biorebsorblable stent. 2018, Tsinghua University.

47. Anagnostopoulos, C.E., M.J.S. Prabhakar, and C.F. Kittle, Aortic dissections and dissecting aneurysms. American Journal of Cardiology, 1972. 30(3): p. 263-73.

48. Wang, S., et al., Short-term curative effect of endovascular stent-graft treatment for aortic diseases in China: a systematic review. Plos One, 2013. 8(8): p. e71012.

49. Jamshidi, P., K. Mahmoody, and P. Erne, Covered stents: a review. International Journal of Cardiology, 2008. 130(3): p. 310-318.

50. Chen, X., Research of Customized Aortic Stent Graft Manufacture. 2017, Tsinghua University.

51. Li, R., Structure analysis of different textile-based artificial vascular. Progress in Textile Science \& Technology, 2007(6): p. 17-17.

52. Wang, F., Desigh and Properties of Branched Graft for Endovascular Prostheses Application. 2014, Donghua University.

53. Wang, L., Design and Process Investigation on a New Type of Thoracic Stent. 2013, Dalian University of Technology.

54. Zhang, L., Fabrication and fundamental research of multi-branched blood vessel scaffolds with multi-layered wall. 2006, Tsinghua University. 